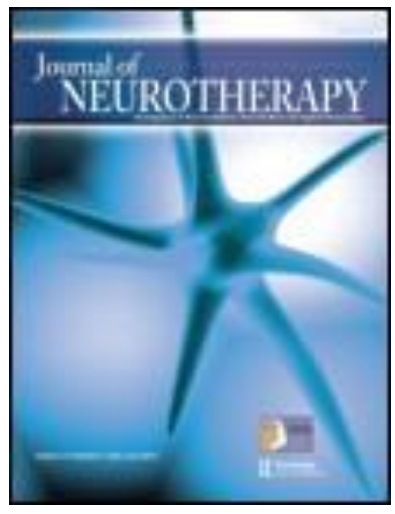

Journal of Neurotherapy: Investigations in Neuromodulation, Neurofeedback and Applied Neuroscience

\title{
Automatic Artifact Detection, Overlapping Windows, and State Transitions
}

David A. Kaiser PhD ${ }^{a} \&$ M. Barry Sterman PhD ${ }^{a}$

${ }^{a}$ Sterman-Kaiser Imaging Laboratory, Bel Air, CA, USA Published online: 20 Oct 2008.

To cite this article: David A. Kaiser PhD \& M. Barry Sterman PhD (2000) Automatic Artifact Detection, Overlapping Windows, and State Transitions, Journal of Neurotherapy: Investigations in Neuromodulation, Neurofeedback and Applied Neuroscience, 4:3, 85-92, DOI: 10.1300/J184v04n03_06

To link to this article: http://dx.doi.org/10.1300/J184v04n03_06

\section{PLEASE SCROLL DOWN FOR ARTICLE}

(c) International Society for Neurofeedback and Research (ISNR), all rights reserved. This article (the "Article") may be accessed online from ISNR at no charge. The Article may be viewed online, stored in electronic or physical form, or archived for research, teaching, and private study purposes. The Article may be archived in public libraries or university libraries at the direction of said public library or university library. Any other reproduction of the Article for redistribution, sale, resale, loan, sublicensing, systematic supply, or other distribution, including both physical and electronic reproduction for such purposes, is expressly forbidden. Preparing or reproducing derivative works of this article is expressly forbidden. ISNR makes no representation or warranty as to the accuracy or completeness of any content in the Article. From 1995 to 2013 the Journal of Neurotherapy was the official publication of ISNR (www. Isnr.org); on April 27, 2016 ISNR acquired the journal from Taylor \& Francis Group, LLC. In 2014, ISNR established its official open-access journal NeuroRegulation (ISSN: 2373-0587;

www. neuroregulation.org).

\section{THIS OPEN-ACCESS CONTENT MADE POSSIBLE BY THESE GENEROUS SPONSORS}

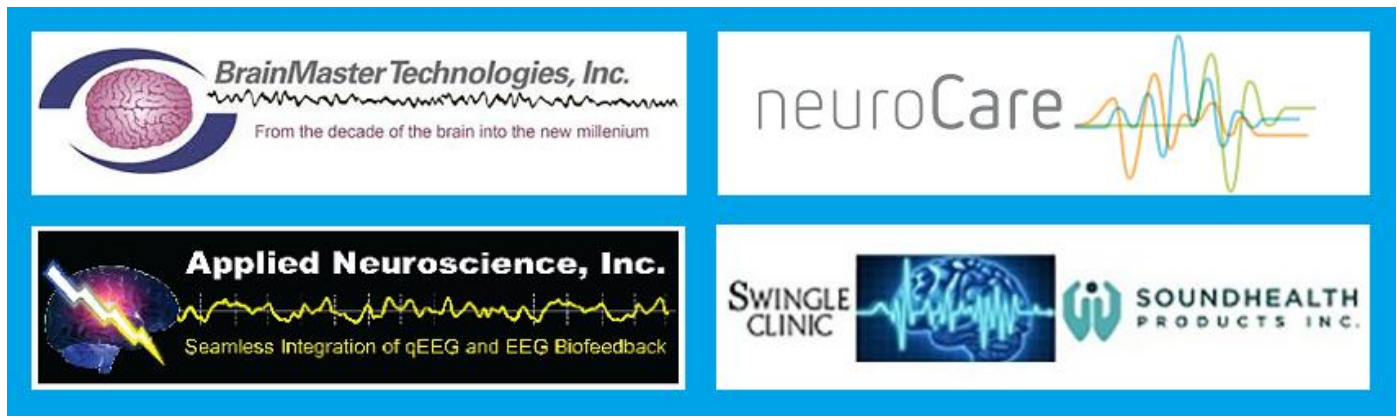




\title{
Automatic Artifact Detection, Overlapping Windows, and State Transitions
}

\author{
David A. Kaiser, $\mathrm{PhD}$ \\ M. Barry Sterman, $\mathrm{PhD}$
}

Earlier laboratory studies of quantitative EEG focused on correlates of human attention and performance disclosed many unresolved technical issues and obstacles. In response to these experiences, Kaiser and Sterman have developed a new QEEG analysis program (SKIL Topometric Software System) designed to address these problems and improve the quality and reliability of evaluations. In this volume, and in a forthcoming series of technical articles, we plan to address these problems and describe the solutions we adopted, as well as some of the new metrics made possible by these solutions.

Artifact Detection. The experienced eye can detect ocular artifacts-eye movements and blinks-with relative ease. The composition of these distinct waveforms, in terms of aberrant amplitude and duration, is noticeably dissimilar from cortical potentials. Eye blinks produce electrical magnitudes up to $800 \mu \mathrm{V}$, dozen of times larger than most cortical potentials. Blinks are gener-

David A. Kaiser and M. Barry Sterman are affiliated with Sterman-Kaiser Imaging Laboratory, Bel Air, CA.

Address correspondence to: David A. Kaiser, PhD, Box 49196, Los Angeles, CA 90049 (E-mail: dakaiser@skiltopo.com).

Journal of Neurotherapy, Vol. 4(3) 2000

Copyright (C) 2000 ISNR. All rights reserved. 
ally slow, lasting up to $400 \mathrm{~ms}$ in duration, although their initial fast components can generate electrical potentials with frequency components of up to about $10.5 \mathrm{~Hz}$, well within the alpha range (Gasser, Stroka \& Mocks, 1985). Eye movements show similar spatial distributions but with lower amplitudes. Classifying EEG signals by waveform characteristics should prove to be useful in automating ocular artifact detection. From our research we developed a series of algorithms, implemented in the SKIL Topometric system, which proved very successful in doing just this (Figure 1). In this system, when artifact is detected, its approximate duration is calculated and marked so that the data segment may be ignored in its entirety during spectral processing.

Given the potential imperfections inherent in any automatic artifacting system, this system is open to manual input and alteration. The sensitivity of the detection algorithms can be altered for each record, the artifact duration calculations can be made more or less conservative, and the actual outputs from the system (the marked data segments) can be lengthened or shortened by hand. Nonetheless, care must be taken when using any automatic routines in QEEG analysis. This waveform classification system, for instance, does not address artifacts generated by muscle discharge and reference problems. The present system supplements, but does not replace, visual inspection of the EEG.

FIGURE 1. An example of the SKIL Topometric Artifact algorithms detecting ocular artifacts in an EEG record. The sensitivity and extent of data loss can be controlled by users as well as the electrode site utilized in the identification process. Data segments containing artifact are marked in the chart by a line at the top of the display.

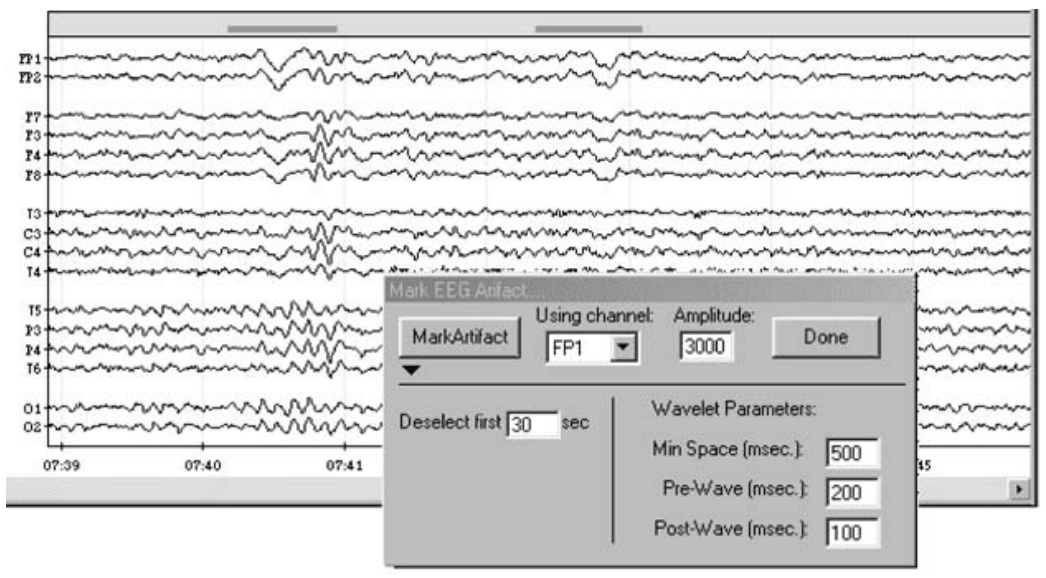


Window Effects. Artifact undermines the reliability and validity of a QEEG evaluation. Artifacts can enter an EEG recording not only at data acquisition but also during later processing. Window functions for Fourier transforms and state transitions are two large sources of potential artifact, neither of which has been thoroughly addressed in the literature. Fortunately, these types of artifact can be minimized with specific strategies and techniques.

Fast Fourier Transforms (FFT), a popular analytical technique, is a powerful and rapid means of identifying frequency components in an EEG signal but it is also a source of subtle yet pervasive artifact. This technique requires that a continuous signal be segmented (into $2^{\mathrm{n}}$ data points), which is likely to produce "sharp edges," non-zero values at the start and end of every segment or epoch. When these epochs are submitted to frequency analysis, the sudden amplitude change between consecutive data points at either edge (from zero to whatever value begins or ends the epoch) will result in erroneous spectral information known as "leakage" (Jervis, Coelho \& Morgan, 1989). Mathematical functions called "windows" or "frames" are commonly applied to data segments to reduce spectral leakage. These functions attenuate data points at either end of an epoch. To taper end points without producing leakage elsewhere within the epoch, all data points in an epoch undergo the same mathematical transformation, usually a gradual and monotonic function. While a tapering function effectively eliminates leakage, it smears or broadens frequency peaks, reducing signal power (Jervis, Coelho, \& Morgan, 1989), and it preferentially samples the epoch's data, an issue rarely discussed in the scientific literature. As shown in Figure 2, EEG events emerging at the center of an epoch dominate the record of that epoch, whereas the same waveforms positioned near either end become nearly invisible, contributing little to spectral analysis.

The use of multiple overlapping windows (i.e., mathematical tapering functions) can remedy the effect of preferential sampling in its entirety (Davidson, Chapman, Chapman \& Henriques, 1990; Sterman, Mann, Kaiser \& Suyenobu, 1994). Unfortunately, this practice has only recently become popular. It should be standard practice for the field. As we discovered, the degree of function overlap does not have to be excessive to remedy sampling effects. The maximum possible overlap is a separate window function begun at each and every data point in a signal. For instance, for a 128-samples-per-second signal segmented into one-second epochs, 128 one-second-window functions per epoch, or $99.2 \%$ overlap, will produce the best possible estimate of spectral density attainable with FFTs. However, a high-quality estimate of spectral density can be achieved with fewer overlapping windows. As shown in Figure 3, the maximum degree of overlap for a 128 -sample rate $(99.2 \%$ or 
FIGURE 2. The effects of a cosine-tapering window on the raw EEG recording are depicted. In all but the bottom trace, an eye blink has been added to a pure $10-\mathrm{Hz}$ signal at the time position noted to the right. Each epoch was submitted to a cosine tapering function to demonstrate how such window functions can augment the contributions of signal components when they occur at the middle of an epoch (625 ms to $1375 \mathrm{~ms}$ ) but diminish their contribution when they occur at either end ( $250 \mathrm{~ms}$ or $1750 \mathrm{~ms})$.

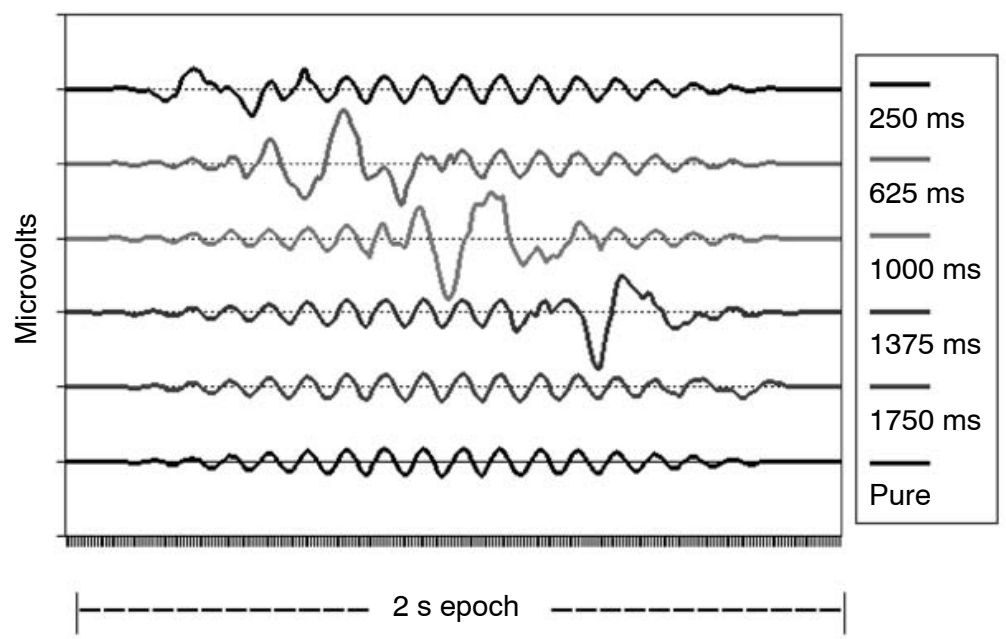

128 window functions per epoch) is well matched by a $75 \%$ overlap (4 windows per epoch).

Notice how poor an estimate of spectral density emerges when overlapping windows are not employed. When window functions do not overlap, spectral values deviate substantially from the optimal spectral estimate (here, $99.2 \%$ overlap). This is not surprising; empirical results merely reflect the calculations involved in generating the window weighting function. When window functions do not overlap, the contribution of data values wax and wane, depending upon their temporal position within the epoch. But when as few as four windows are used to estimate spectral density, an almost perfectly straight weighting function is derived (see Figure 4). A straight weighting function means that every data value in the epoch is weighted equally, which is of course highly desirable. As shown in Table 1, an analysis of three degrees of overlap confirms this observation. Table 1 also suggests that when 
FIGURE 3. Mean effect of overlapping windows (none, 4 per epoch, 128 per epoch) on 8-12 Hz spectral means during 60 seconds of an eyes closed baseline $(n=4)$.

Windowing Effect

Eyes Closed $(\mathrm{Pz}, 8-12 \mathrm{~Hz}, \mathrm{n}=4)$

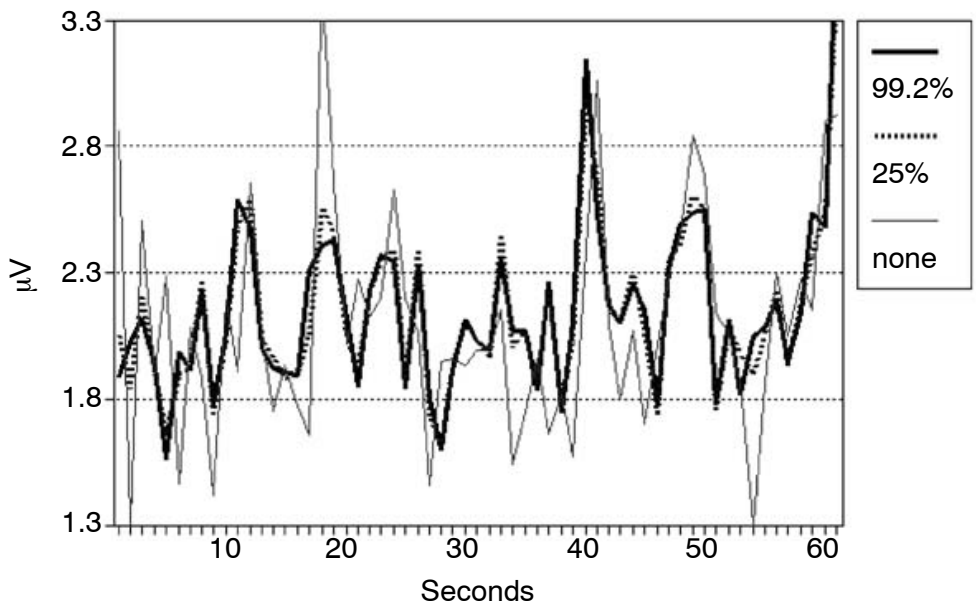

no overlap is performed, a false interaction between condition and frequency band may emerge.

Collectively, these results suggest that as few as four window functions per epoch (i.e., 75\% overlap) provide an excellent estimate of spectral density, satisfying a compromise between calculational complexity and statistical rigor. (The SKIL Topometric system employs $75 \%$ overlap in all of its spectral analyses.)

State Transitions. Artifact can also creep into one's results from poor control of a subject's mental state during recordings. In our laboratory studies we discovered that state transitions associated with the commencement of an EEG data file can distort the initial portion of that file due to either slow transitions or abrupt shifts in attention (see Figure 5). This effect was particularly strong for resting (baseline) conditions and less important during task recordings, probably due to the immediate and continuous constraints most tasks place on an individual's mental state. Fortunately, this form of state instability can be easily corrected. By omitting the first 30 seconds from analysis or by waiting 30 seconds before initiating data collection such state transitions may be avoided. The former approach is readily automated in software. In fact during the SKIL database creation, the first 30 seconds of every baseline recording (eyes closed and eyes open resting) were omitted 
FIGURE 4. Derived weighting functions for $0 \%$ - and $75 \%$-overlapping cosinetapering windows are shown. Each weighting function (in red) is simply the average of the curving lines below it. For clarity's sake, a constant has been added to each weighting function so that this average does not coincide with the windows. Note how data points are sampled (weighted) preferentially in the top figure, but when four evenly spaced cosine-tapering windows are used, preferentially weighting all but vanishes (a flat line average). With overlapping windows, initial data points are ignored during analysis unless each point appears in all available windows (here, the first $750 \mathrm{~ms}$, those under the saw tooth pattern, are not included in later analysis).

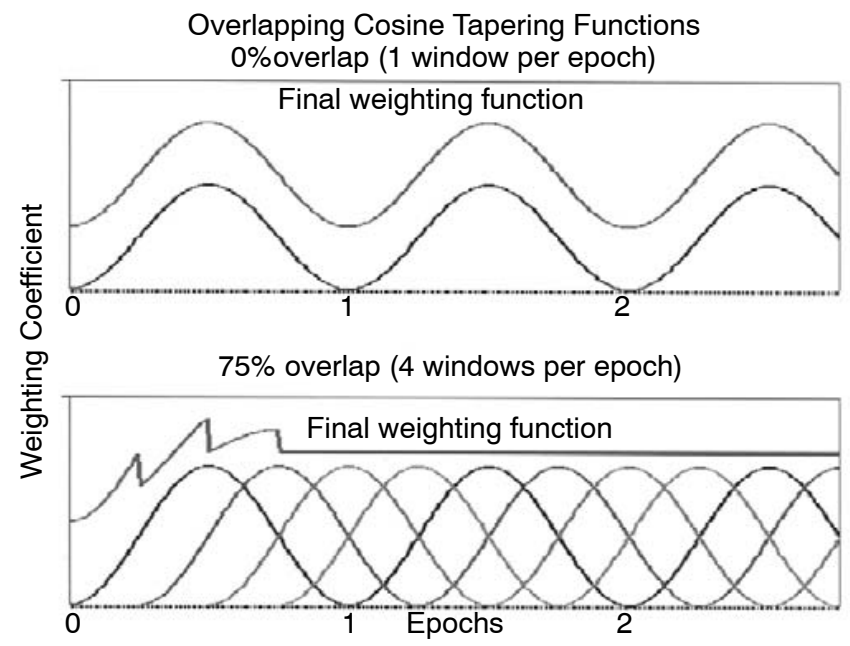

TABLE 1. Mean correlation between three degrees of overlap in three frequency bands averaged across 19 sites for eyes closed (EC), eyes open (EO), and block design (BD) conditions $(n=4)$.

\begin{tabular}{lcccc} 
& & $1-4 \mathrm{~Hz}$ & $8-12 \mathrm{~Hz}$ & $12-18 \mathrm{~Hz}$ \\
\hline EC & $128 \times 1$ & 0.41 & 0.43 & 0.50 \\
EO & $128 \times 1$ & 0.33 & 0.23 & 0.42 \\
BD & $128 \times 1$ & 0.45 & 0.38 & 0.28 \\
EC & $128 \times 4$ & 0.95 & 0.96 & 0.96 \\
EO & $128 \times 4$ & 0.95 & 0.95 & 0.96 \\
BD & $128 \times 4$ & 0.96 & 0.95 & 0.95 \\
EC & $4 \times 1$ & 0.60 & 0.61 & 0.66 \\
EO & $4 \times 1$ & 0.53 & 0.44 & 0.58 \\
BD & $4 \times 1$ & 0.63 & 0.59 & 0.50
\end{tabular}


FIGURE 5. Three replications of an eyes closed resting conditions (50 seconds smoothed, $\mathrm{n}=20$ ) demonstrate the state transition of each replication during the first 30 seconds or so.

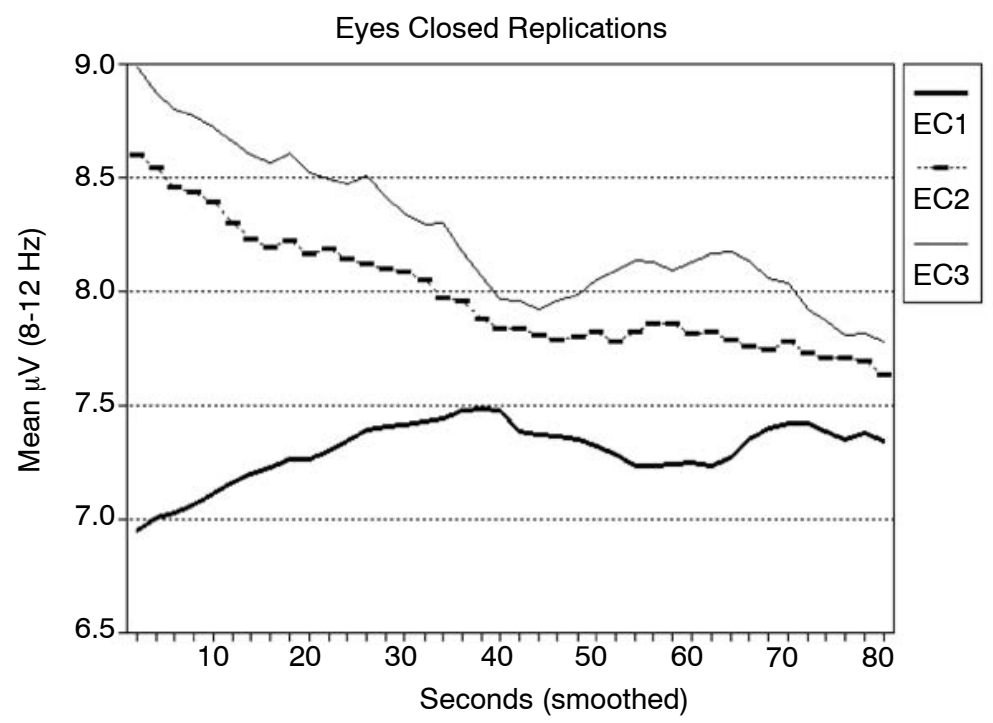

from mean calculations. Comparison to this database requires a similar omission, either during acquisition or analysis.

Additional Issues. Time-of-day effects, or chronobiological influences produce an important source of significant processing artifact in QEEG analysis. This problem has been grossly disregarded in the clinical QEEG literature. The effects of circadian, semidian, and ultradian rhythms on the EEG will be discussed in a future article, along with additional issues critical to QEEG evaluation. These include the need for challenge or task conditions when evaluating individuals with ADHD and other disorders, as well as the promise of event-related processing in this field. Further information about the SKIL Topometric system is available online (www.skiltopo.com).

\section{REFERENCES}

Davidson, R.J., Chapman, J.P., Chapman, L.J., \& Henriques, J. (1990). Asymmetrical brain electrical activity discriminates between psychometrically matched verbal and spatial cognitive tasks. Psychophysiology, 27, 528-543.

Gasser, T., Stroka, L., \& Mocks, J. (1985). The transfer of EOG activity into the EEG for eyes open and closed. Electroencephalography and Clinical Neurophysiology, 34, 212-216. 
Jervis, B.W., Coelho, M., \& Morgan, G.W. (1989). Spectral analysis of EEG responses. Medical and Biological Engineering and Computing, 27, 230-238.

Sterman, M.B., Mann, C.A., Kaiser, D.A., \& Suyenobu, B.Y. (1994). Multiband topographic EEG analysis of a simulated visuomotor aviation task. International Journal of Psychophysiology, 16, 49-56.

for faculty/professionals with journal subscription recommendation authority for their institutional library . . .

If you have read a reprint or photocopy of this article, would you like to make sure that your library also subscribes to this journal? If you have the authority to recommend subscriptions to your library, we will send you a free sample copy for review with your librarian. Just fill out the form below-and make sure that you type or write out clearly both the name of the journal and your own name and address.

( ) Yes, please send me a complimentary sample copy of this journal:

(please write in complete journal title here-do not leave blank)

I will show this journal to our institutional or agency library for a possible subscription.

The name of my institutional/agency library is:

NAME:

INSTITUTION:

ADDRESS:

CITY: STATE: ZIP:

Return to: Sample Copy Department, The Haworth Press, Inc., 10 Alice Street, Binghamton, NY 13904-1580 